\title{
CUATRO NUEVOS REGISTROS Y UNA NUEVA ESPECIE DE CUCURBITACEAE PARA LA FLORA DE OAXACA ${ }^{1,2}$
}

\author{
Rafael lira SaAde y Rafael Torres Colin \\ Herbario Nacional de México \\ Instituto de Biologia U.N.A.M. \\ Apdo. Postal 70-233 \\ Delegación Coyoacán, 04510 México, D.F.
}

\begin{abstract}
RESUMEN
Cuatro especies de Cucurbitaceae [ Tecunumania quetzalteca Standley \& Steyermark, Cionosicyos macranthus (Pittier) C. Jeffrey, Peponopsis adhaerens Naudin y Chalema synanthera Dieterle] se registran por primera vez para la flora del estado de Oaxaca (la última además para Guerrero) y se describe una nueva especie de Parasicyos Dieterle.
\end{abstract}

\section{ABSTRACT}

Four species of Cucurbitaceae [Tecunumania quetzalteca Standley \& Steyermark, Cionosicyos macranthus (Pittier) C. Jeffrey, Peponopsis adhaerens Naudin and Chalema synanthera Dieterle] are reported for the first time for the flora of Oaxaca (the latter also for Guerrero) and a new species of Parasicyos Dieterle is described.

Durante la revisión de ejemplares de la familia Cucurbitaceae recolectados en conexión con el proyecto Flora de Oaxaca del Herbario Nacional de México, se lograron identificar algunos que resultaron corresponder a cuatro nuevos registros para la flora de dicho estado y a una nueva especie.

\section{Nuevos Registros}

Tecunumania quetzalteca Standley \& Steyermark. Esta especie fue descrita de Finca Vergel en San Marcos, Guatemala (Standley \& Steyermark, 1944) y hasta ahora sólo se conocía del estado de Chiapas, México, así como de Guatemala y Costa Rica (Dieterle, 1976). En Oaxaca, Tecunumania quetzalteca fue encontrada en una selva alta perennifolia

1 Trabajo realizado con apoyo económico del proyecto "Flora de Oaxaca" del Herbario Nacional de México (MEXU), del Instituto de Biologia, U.N.A.M.

2 El trabajo de campo desarrollado en 1990 por el primer autor, fue financiado por el International Board for Plant Genetic Resources, a' través del proyecto "Estudios Taxonómicos y Ecogeográficos de las Cucurbitaceae de Latinoamérica", a su cargo en el Herbario Nacional de México. 
bien conservada, prosperando sobre suelos arenosos con abundantes rocas. Se encontró asociada con Dorstenia sp., Chamaedorea sp., Esenbeckia sp., Lonchocarpus sp. Heliocarpus sp. y Dracaena sp.

Aunque Dieterle (1976) señala que en apariencia general (principalmente por sus hojas y sus vistosas flores amarillas) $T$. quetzalteca tiene cierto parecido con Luffa aegyptiaca Miller, difiere de esta última por presentar flores estaminadas solitarias (en racimos en $L$. aegyptiaca), con las anteras fusionadas y los filamentos conniventes (estambres completamente libres en L. aegyptiaca). Otros caracteres distintivos de esta especie son la presencia de una densa agrupación de pelos en el ápice del peciolo y los sépalos angostos y usualmente más largos que el cáliz.

Ejemplar examinado: Oaxaca. Mpio. de Santa María Jacatepec. Distrito de Tuxtepec. Predio El Aguila, al O de San Agustín entrando por La Reforma, $28 \mathrm{~km}$ al SO de Tuxtepec, carr. a Matias Romero. $17^{9} 50^{\prime} \mathrm{N} ; 9^{2} 06^{\prime} \mathrm{O}$. Selva alta perennifolia. Altitud, $550 \mathrm{~m}$. Enredadera; flor amarilla. 19.I.1988. R. Torres 10995 (MEXU).

Cionosicyos macranthus (Pitt.) C. Jeffrey. El área de distribución conocida hasta ahora para esta especie abarcaba los estados de Veracruz, Tabasco, Chiapas y Campeche en el sur de México y, en Centroamérica desde Belice hasta Panamá (Jeffrey, 1971, 1990 com. pers.; Lira, 1985; Pittier, 1910; Wunderlin, 1978). En Oaxaca, Cionosicyos macranthus prospera en la región norte del estado, en una zona muy cercana a los límites con el estado de Veracruz.

Entre las características distintivas de esta especie están sus vistosas flores de color blanco verdoso y sus frutos subglobosos a elípticos, de 6-7 cm de diámetro, de color verde a pardo claro con rayas de un tono de verde más obscuro. La pulpa de ios frutos es carnosa y suave, tornándose de color anaranjado y sabor ligeramente dulce al madurar, con numerosas semillas angostamente oblongas, de color pardo obscuro a negro, con el hilo ligeramente oblicuo.

Ejemplar examinado: Oaxaca. Mpio. de San Lucas Ojitlán. La Raya, carretera para el pueblo El Zapotal. Acahual derivado de selva alta perennifolia secundaria. Altitud, 550 $\mathrm{m}$. Suelos rojos rocosos. Abundancia regular. Hierba trepadora, $4 \mathrm{~m}$, anual. Fruto verde, anaranjado por dentro, semillas negras. Nombre Local: "fruto de pepe". 22.I.1989. J.I. Calzada 14263 (MEXU).

Chalema synanthera Dieterle. Esta especie fue descrita de localidades cercanas a Apatzingán, Michoacán y Chamela, Jalisco (Dieterle, 1980), en donde crece en selva baja o mediana caducifolia o subcaducifolia a menos de $300 \mathrm{~m}$ s.n.m. En Oaxaca, fue recolectada en sitios cercanos a las costas de Huatulco, en una selva mediana caducifolia perturbada con Lonchocarpus phaseolifolius, Lasiacis ruscifolia, Cyperus hermaphroditus, Rivina humilis, etc., en aititudes entre 150 y $300 \mathrm{~m}$. También fue registrada por primera vez para el estado de Guerrero.

Chalema synanthera es una planta con tallos delgados, hojas delicadas y herbáceas y flores blancas diminutas dispuestas en panículas. En toda su área de distribución florece y fructifica entre julio y diciembre. El carácter monoico de esta especie, junto con la estructura de sus estambres (filamentos fusionados con 5 anteras individualizadas, aunque unidas formando una especie de anillo), permiten distinguirla del resto de la subfamilia Zanonioideae. 
Ejemplares examinados: Oaxaca. Mpio. de Santa María Huatulco. Distrito de Pochutla. Bahía de Tangolunda. Bosque tropical caducifolio en costa. Planta herbácea, trepadora, flor blanca, abundante. 11.X.1984. R. López 149 (MEXU); $1.2 \mathrm{~km}$ al sur de la desviación a Bahía dé Santa Cruz; la desviación está a 15.2 km al SO de Copalita. Selva mediana, $150 \mathrm{~m}$ s.n.m. Enredadera con flores verdosas. 30.VII.1984. R. Torres \& C. Martinez 5766 (MEXU); $1 \mathrm{~km}$ al sur de la desviación a Sta. Cruz Huatulco. Distrito de Pochutla. Selva mediana caducifolia. Suelo pedregoso. Altitud, $200 \mathrm{~m}$. Hierba trepadora con flores blancas. 25.IX.1984. R. Torres \& E. Cabrera 6236 (MEXU). Guerrero. Al este del mirador del Parque Ecológico "La Vainilla" en el cerro "Bolonche". Mpio. de Zihuatanejo. Altitud, $490 \mathrm{~m}$. Bosque tropical subcaducifolio (ladera norte del cerro). Enredadera, flor blanca. 27.X.1989. C. Gallardo et al. 207 (FCME, MEXU).

Peponopsis adhaerens Naudin. Esta especie endémica de México, fue descrita en 1860 por Ch. Naudin a partir de ejemplares cultivados en París, probablemente procedentes del noreste de México (Cogniaux, 1881; Jeffrey, 1971). Se trata de una planta muy poco conocida; sus frutos y semillas fueron descritos en 1985, y hasta ahora solamente se habia registrado de un área relativamente pequeña en el norte de Veracruz y zonas aledañas de los estados de Puebla, San Luis Potosí e Hidalgo (Nee, 1990), en donde habita en selvas bajas caducifolias y medianas subperennifolias. En Oaxaca esta especie fue encontrada con flores estaminadas y frutos, creciendo en un bosque mesófilo de montaña con Podocarpus, Clethra, Palicourea, Smilax y numerosas epifitas de las familias Orchidaceae, Bromeliaceae y Piperaceae, a elevaciones entre 1400 y 1700 m s.n.m.

Peponopsis adhaerens presenta flores solitarias, muy vistosas de color amarillo. Las estaminadas tienen los sépalos notablemente agrandados, los cuales en botón alcanzan a cubrir totalmente a la corola. Sus frutos son globosos, lisos, de color verde olivo, de 8-10 cm de diámetro; la pulpa se encuentra formando una especie de compartimentos o pequeñas cámaras cartilaginosas, es de color blanquecino a amarillo pálido y tiene un sabor ligeramente dulce; las semillas son muy numerosas (150-200 por cada fruto), ovadooblongas, comprimidas, grisaceas, $7-8 \times 4-5 \mathrm{~mm}$, cada una ocupando una de las cámaras de la pulpa.

Ejemplar examinado: Oaxaca. Distrito de Tehuantepec. Cerro Selva del Aserradero, al E del crucero Guadalupe (12.5 km al NE de Santiago Lachiguiri). $16^{\circ} 43^{\prime} \mathrm{N}, 9^{\circ} 30^{\prime} \mathrm{O}$. Bosque mesófilo de montaña. Altitud, 1400-1700 m. Enredadera de flores amarillas y frutos verdes. 9.V.1991. R. Torres \& A. Campos V. 13947 (MEXU).

\section{Nueva Especie de Parasicyos}

Parasicyos pertenece a la subtribu Sicyinae, tribu Sicyeae (Jeffrey, 1990). Fue propuesto por Dieterle (1975) para ubicar a $P$. maculatus, única especie hasta ahora conocida de este género, la cual fue descrita a partir de ejemplares provenientes de Alta Verapaz, Guatemala (Williams et al. 40205; Isotipo: $\mathrm{MICH}$ !).

De acuerdo con Dieterle (1975), los taxa más cercanos a Parasicyos son Microsechium y Sicyos, con los cuales comparte la ausencia de nectarios en la base del cáliz y la presencia de flores estaminadas y frutos agrupados sobre un pedúnculo común. 
Parasicyos difiere de Microsechium por presentar flores estaminadas pentámeras (tetrámeras en Microsechium), los filamentos completamente fusionados (parcialmente libres con ramificaciones patentes en Microsechium) y las anteras coherentes pero libres hacia el ápice y sésiles sobre la columna de los filamentos (completamente libres en el ápice de las delgadas ramificaciones de los filamentos en Microsechium). La diferencia más importante respecto a Sicyos corresponde a los frutos, los cuales en Parasicyos son de tamaño medio, carnosos, inermes y conspicuamente maculados, mientras que en Sicyos son secos, comparativamente pequeños, con las paredes delgadas, frecuentemente espinosos y/o tuberculados y no maculados.

Las características antes mencionadas para las flores estaminadas y los frutos de $P$. maculatus, están claramente representadas en la planta que se propone a continuación como nueva especie.

Parasicyos dieterleae Lira et Torres sp. nov.

A P. maculato Dieterle foliis subtus pubescentibus vel hispidulis, lobulo medio elliptico ad basim angustato, cirrhis bifidis vel raro simplicibus, inflorescentiis masculis racemosis, pedicellis masculis brevioribus, sepalis lanceolatis, corolla partim partita, petalis grandioribus, pedunculis fructiferis parvioribus et fructibus subglobosis vel obovoideis parvioribus differt.

Herbácea vigorosa, trepadora, monoica, con raices masivas perennes. Tallos sulcados, esparcidamente pubescentes a densamente puberulentos en los nudos, glabrescentes. Hojas alternas, ovado-cordadas, coriáceas cuando frescas a firmemente papiraceas o cartaceas al secar, $6-14.5 \mathrm{~cm}$ de largo, $5-15.5 \mathrm{~cm}$ de ancho, 3-lobadas, los lóbulos elípticos, acuminados, el central más grande que los laterales, angostado hacia la base, los laterales ascendentes y ligeramente lobados o auriculados en la base, base cordada, márgenes denticulados; superficie adaxial de color verde intenso, escabrosopustulada; superficie abaxial de color verde pálido, densamente pubescentes cuando jóvenes a esparcidamente hispídulas sobre las venas con la edad, las venas resaltadas, amarillentas a parduscas; pecíolo sulcado, $1-3.5 \mathrm{~cm}$ de largo, densamente pubescente a puberulento. Zarcillos simples a más comunmente blfidos, sulcados, esparcidamente puberulentos a glabros. Flores estaminadas en inflorescencias racemosas, frecuentemente con ramificaciones muy breves ( $<2 \mathrm{~cm}$ de largo), $8.5-22 \mathrm{~cm}$ de largo, pedúnculo usualmente mucho más largo que el raquis; pedicelos angulosos, puberulentos, 1-5 mm de largo; cáliz anchamente campanulado, 3-4.5 $\mathrm{mm}$ de largo, 2.5-3 $\mathrm{mm}$ de ancho, esparcidamente puberulento; sépalos 5 , lanceolados, 0.9-1.5 (-2) $\mathrm{mm}$ de largo, glabros, patentes principalmente en botón; corola blanco verdosa, rotada, 5-dividida hasta más o menos $3 / 4$ de su longitud total; pétalos triangulares, obtusos, $2-4.5(-5) \mathrm{mm}$ de largo y casi otro tanto de ancho en la base, esparcidamente pilosos por fuera, densamente papilosopuberulentos por dentro; filamentos fusionados formando una columna corta y engrosada, 0.6-0.9 mm de largo; anteras 3, flexuoso-conduplicadas, coherentes para formar una estructura subglobosa, aunque más o menos libres hacia el ápice. Flores pistiladas usualmente en la misma axila que las estaminadas, 2-3 subsésiles sobre un pedúnculo común; pedúnculo engrosado, $2-7 \mathrm{~mm}$ de largo, pubescente, glabrescente con la edad; 
ovario subgloboso a ovoide, 0.8-3.5 mm de largo, 0.5-2.0 mm de ancho, pubescente, glabrescente con la edad, unilocular, un solo óvulo, péndulo; perianto como en las estaminadas pero de tamaño más reducido; estilo corto, $1 \mathrm{~mm}$ de largo, engrosado; estigmas 3, subglobosos. Frutos 1-3 subsésiles sobre un pedúnculo común de 7-15 (-20) $\mathrm{mm}$ de largo, subglobosos a obovoides, (9-) 13-23 (-28) mm de largo, 8-20 mm de diámetro en su porción más ancha, verdes y concoloros cuando jóvenes, tornándose al madurar de color verde claro maculado de blanco o verde más pálido, y pardo claro maculado de pardo obsćuro al secar; pulpa fibrosa de sabor muy amargo; una sola semilla en posición vertical, ovoide, testa lisa, muy rigida y engrosada.

TIPO: Oaxaca. Mpio. de Teposcolula. Distrito de Teposcolula. Cerro El Peñasco al S de Teposcolula. Bosque de encino-pino. Elevación 2200-2550 m s.n.m. Enredadera, fruto verde, flor verde. R. Torres \& M.L. Torres 12318 (9.IX.1988) (flores estaminadas, flores pistiladas, frutos inmaduros y maduros) (Holotipo: MEXU, Isotipos: por distribuirse).

Material adicional examinado: Oaxaca. Pie del Cerro La Falda, a $1 \mathrm{~km}$ al $\mathrm{O}$ de San Pedro y San Pablo Teposcolula. Distrito de Teposcolula. Altitud, 2000 m. M. Sousa, C. Ramos \& O. Téllez 5958. 22.VIII.1976 (flores estaminadas, flores pistiladas y frutos inmaduros) (MEXU); Río Teposcolula, $500 \mathrm{~m}$ al $\mathrm{S}$ del poblado. Distrito de Teposcolula, Mixteca Alta. Vegetación riparia con Alnus acuminata, Salix sp., Fraxinus sp. y Prunus serotina. Suelo pardo claro, limoso. Altitud, 2140 m. A. Garcia M. 546. 17.VII.1981 (flores estaminadas y flores pistiladas) (MEXU, XAL); Cerro El Peñasco, $1 \mathrm{~km}$ al O de Teposcolula. Distrito de Teposcolula. Matorral esclerofilo con Rhus, Quercus, Juniperus, Amelanchier. Altitud, $2340 \mathrm{~m}$. Suelo negro, escaso, derivado de calizas. A. García M., D. Lorence \& D. Frame 1704. 26.VII.1985 (flores estaminadas, flores pistiladas y frutos inmaduros) (MEXU). Distrito de Teposcolula. Mpio. Teposcolula. $1 \mathrm{~km}$ al E de Teposcolula, rumbo al Cerro Peñasco, a orillas del río Teposcolula. Vegetación riparia con Fraxinus, Populus, Alnus. Altitud, 2150 m. R. Lira \& J.C. Soto 1103. 18.X.1990 (frutos maduros) (MEXU); Distrito de Teposcolula. Poblado Guadalupe Tixá, (desviación sobre la carretera Teposcolula-Tlaxiaco, aproximadamente $4 \mathrm{~km}$ al $\mathrm{S}$ de Teposcolula). Vegetación secundaria de Bosque de Pinus. Altitud, 2150 m. Suelos calizos. R. Lira \& J.C. Soto 1106. 18.X.1990 (frutos maduros) (MEXU).

Hasta la fecha, $P$. dieterleae sólo se conoce del área de la localidad tipo en el Distrito de Teposcolula, Oaxaca, México. Prospera en vegetación riparia con Alnus, Salix, Fraxinus y Prunus, en matorral esclerófilo con Rhus, Quercus y Juniperus y en vegetación secundaria de bosque de Pinus o Pinus-Quercus, a altitudes entre 2150 y $2550 \mathrm{~m}$, en suelos limosos y derivados de calizas.

Parasicyos dieterleae difiere de $P$. maculatus, en la forma de los lóbulos de las hojas (sobre todo del central), el indumento de las mismas, principalmente en la superficie abaxial, la división de los zarcillos, el tipo de inflorescencia estaminada y las dimensiones de las estructuras florales, asi como también en la forma y tamaño de los frutos (Cuadro 1). Aunque la comparación de las flores pistiladas de ambas especies es deseable, ésta no ha sido posible por no contar con ejemplares o información al respecto para $P$. maculatus. 


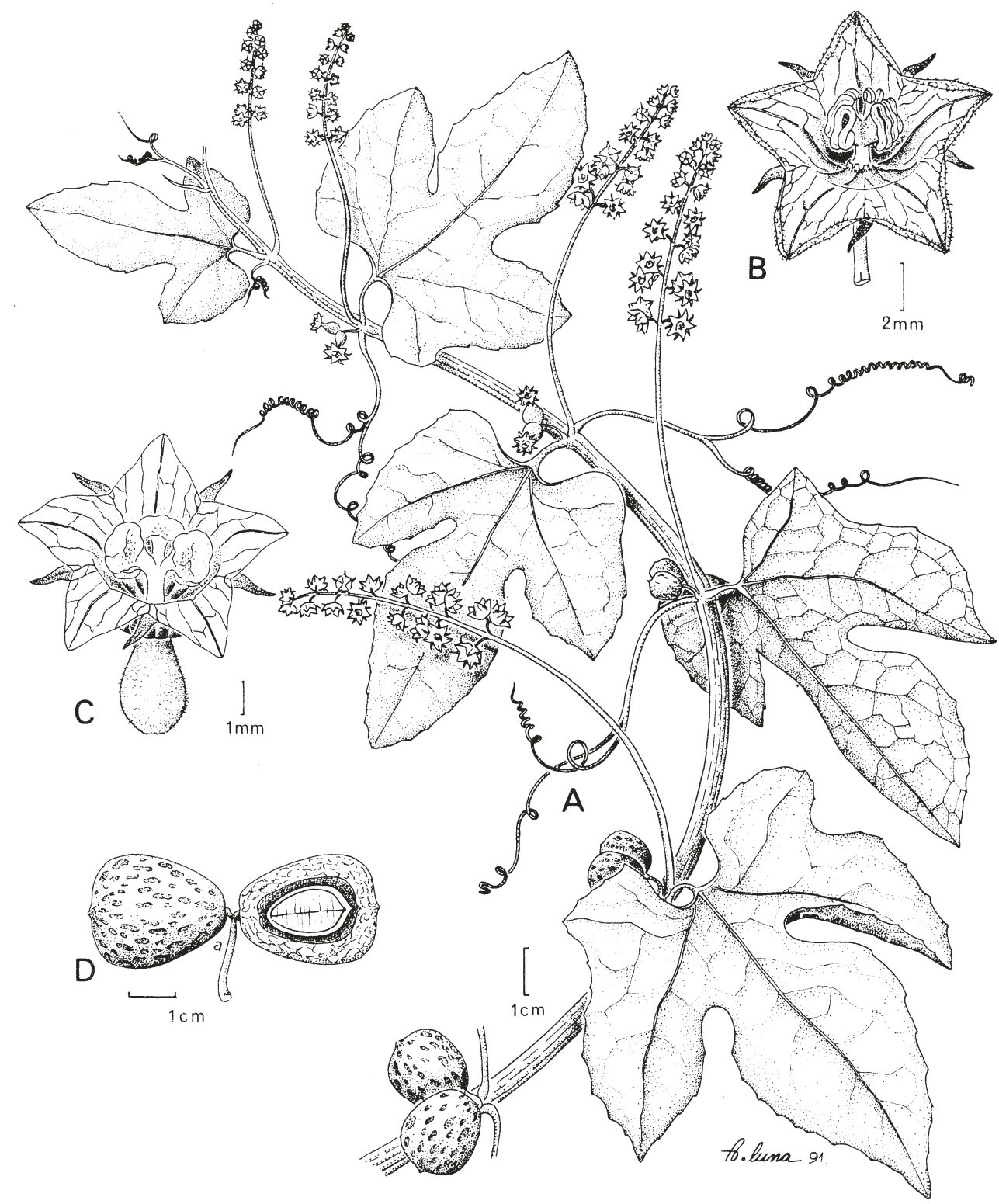

Fig. 1. Parasicyos dieterleae Lira et Torres. a) hábito, b) detalle de la flor estaminada mostrando la estructura de los estambres, c) flor pistilada desprovista de parte del perianto para mostrar la estructura del estilo y los estigmas, d) corte longitudinal del fruto maduro. Basada en los ejemplares Torres \& Torres 12318, Sousa et al. 5958 y Lira \& Soto $1103,1106$. 
A las diferencias morfológicas incluidas en el cuadro 1, se pueden adicionar las diferencias fenológicas observadas en el campo y en el material disponible. Parasicyos dieterleae florece y fructifica entre junio y noviembre, y a partir de entonces sus tallos y hojas se secan por completo para rebrotar en la época lluviosa, mientras que el ejemplar revisado de $P$. maculatus indica que a finales de enero la planta produce frutos y aún presenta flores estaminadas.

Cuadro 1. Comparación de las características morfológicas de Parasicyos dieterleae y $P$. maculatus.

\begin{tabular}{|c|c|c|}
\hline CARACTERISTICAS & $P$. dieterleae & P. maculatus \\
\hline Hojas indumento & $\begin{array}{l}\text { superficie adaxial } \\
\text { escabroso-pustulada, } \\
\text { la abaxial pubescen- } \\
\text { te a hispidula so- } \\
\text { bre las venas }\end{array}$ & $\begin{array}{l}\text { superficie adaxial } \\
\text { diminutamente } \\
\text { escabrosa, la } \\
\text { abaxial glabra }\end{array}$ \\
\hline $\begin{array}{l}\text { Lóbulo central de las } \\
\text { hojas }\end{array}$ & $\begin{array}{l}\text { elíptico, acuminado, } \\
\text { angostado hacia } \\
\text { la base }\end{array}$ & $\begin{array}{l}\text { triangular, acuminado, } \\
\text { ensanchado en } \\
\text { la base }\end{array}$ \\
\hline Peciolos largo $(\mathrm{cm})$ & $1-3.5$ & $2-8$ \\
\hline Zarcillos & $\begin{array}{l}\text { bífidos,ocasional- } \\
\text { mente simples }\end{array}$ & trífidos \\
\hline $\begin{array}{l}\text { Inflorescencia } \\
\text { estaminada }\end{array}$ & $\begin{array}{l}\text { racimos,ocasional- } \\
\text { mente con algunas } \\
\text { ramificaciones muy } \\
\text { breves }(<2 \mathrm{~cm})\end{array}$ & panículas \\
\hline $\begin{array}{l}\text { Pedicelos estaminados } \\
\text { largo }(\mathrm{mm})\end{array}$ & $1-5$ & $5-9$ \\
\hline Sépalos forma & lanceolados & triangular dentiformes \\
\hline Sépalos largo $(\mathrm{mm})$ & $0.9-1.5$ & $0.3-0.5$ \\
\hline Corola división & $\begin{array}{l}\text { 5-dividida hasta ca. } \\
3 / 4 \text { de su longitud } \\
\text { total }\end{array}$ & totalmente 5 -dividida \\
\hline Pétalos tamaño $(\mathrm{mm})$ & $2-4.5(-5) \times 2-4.5$ & $1.2-2.5 \times 0.4-2$ \\
\hline $\begin{array}{l}\text { Pedúnculo frutos } \\
\text { largo }(\mathrm{mm})\end{array}$ & $7-20$ & $35-50$ \\
\hline Frutos forma & $\begin{array}{l}\text { subglobosos a obovoi- } \\
\text { des }\end{array}$ & elípticos \\
\hline Frutos largo $(\mathrm{mm})$ & (9-) $13-23(-28)$ & $30-45$ \\
\hline
\end{tabular}


El nombre de esta especie está dedicado a la Dra. Jennie V. Dieterle (Universidad de Michigan), por sus importantes contribuciones al conocimiento de la familia Cucurbitaceae.

Algunos campesinos entrevistados por el primer autor en los poblados de Teposcolula y Guadalupe Tixá, indicaron que Parasicyos dieterleae es conocida en la región con nombres que aluden a las características de sus frutos, principalmente por su similitud con los de otras especies de la familia Cucurbitaceae, como "chayotillo", "chilacayotito" y "bellota", además del nombre "tindú" de origen mixteco. También se indicó que una infusión de las hojas y la raíz es empleada para eliminar los piojos.

\section{AGRADECIMIENTOS}

Se agradece a Fernando Chiang, Oswaldo Téllez, Patricia Dávila, Ma. del Rosario Garcia y Héctor Hernández (Departamento de Botánica, Instituto de Biología, U.N.A.M.), así como a los revisores anónimos del Comite Editorial de Acta Botánica Mexicana por sus valiosos comentarios y sugerencias. A Albino Luna (Instituto de Biología, U.N.A.M.) por la realización del dibujo de Parasicyos dieterleae y a Abisal García por proporcionar material fotográfico para elaborar dicha ilustración. Fernando Chiang colaboró además con la diagnosis en latín de $P$. dieterleae.

Un agradecimiento especial es para el Dr. William Anderson, Director del Herbario $\mathrm{MICH}$, por permitirnos consultar el isotipo de Parasicyos maculatus, al Dr. Charles Jeffrey (Kew Royal Botanic Gardens) por compartir con nosotros datos inéditos de su revisión de las Cucurbitaceae de Nicaragua, y para Claudia Hernández, estudiante de la Facultad de Ciencias, de la Universidad Nacional Autónoma de México, por proporcionarnos su ejemplar de Chalema synanthera del estado de Guerrero.

\section{LITERATURA CITADA}

Cogniaux, A. 1881. Cucurbitacées. In: De Candolle, A. y C. De Candolle. Monographiae Phanerogamarum 3: $325-951$.

Dieterle, J. V. A. 1975. Parasicyos maculatus a new species and genus of Cucurbitaceae from Guatemala. Phytologia 32: 289-290.

Dieterle, J. V. A. 1976. Cucurbitaceae. In: Nash, D.L. (ed.). Flora of Guatemala. Fieldiana Bot. 24 (XI, 4): 306-395.

Dieterle, J. V. A. 1980. Two new Cucurbitaceae from Mexico. Contr. Univ. Mich. Herb. 14: 69-73.

Jeffrey, C. 1971. Further notes on Cucurbitaceae. II. The tribe Cucurbiteae. Kew Bull. 15: 337-371.

Jeffrey, C. 1990. An outline classification of the Cucurbitaceae. In: Bates, D.M., R.W. Robinson \& C. Jeffrey (Eds.). Biology and Utilization of the Cucurbitaceae. Comstock, Cornell University Press. Ithaca, New York. pp. 449-463.

Lira, R. 1985. Notas del Herbario XAL. IV. Nuevos registros de Cucurbitaceae para la Flora Yucatanense. Biotica 10(3): 297-299.

Nee, M. 1990. The domestication of Cucurbita (Cucurbitaceae). Econ. Bot. 44(3): 56-68.

Pittier, H. 1910. New or noteworthy plants from Colombia and Central America. Contr. U.S. Natl. Herb. 13: 93-132. 
Lira y Torres: Nuevos Registros y Una Nueva Especie de Cucurbitaceae de Oaxaca

Standley, P. C. \& J. C. Steyermark. 1944. Studies of Central American plants. IV. Field Mus. Nat. Hist., Bot. Ser. 23: 31-109.

Wunderlin, R. P. 1978. Cucurbitaceae. In: Woodson, R. E. et al. (eds.). Flora of Panama. Ann. Missouri Bot. Gard. 65: 285-366. 